

\title{
A multi-agent knowledge model for SMEs mechatronic supply chains.
}

\author{
Jihène Tounsi, Georges Habchi, Julien Boissiere, Selma Azaiez
}

\section{To cite this version:}

Jihène Tounsi, Georges Habchi, Julien Boissiere, Selma Azaiez. A multi-agent knowledge model for SMEs mechatronic supply chains.. Journal of Intelligent Manufacturing, 2012, 23 (2647-2665), pp. 2647-2665. 10.1007/s10845-011-0537-1 . hal-00627502

\section{HAL Id: hal-00627502 \\ https://hal.univ-smb.fr/hal-00627502}

Submitted on 8 Jan 2013

HAL is a multi-disciplinary open access archive for the deposit and dissemination of scientific research documents, whether they are published or not. The documents may come from teaching and research institutions in France or abroad, or from public or private research centers.
L'archive ouverte pluridisciplinaire HAL, est destinée au dépôt et à la diffusion de documents scientifiques de niveau recherche, publiés ou non, émanant des établissements d'enseignement et de recherche français ou étrangers, des laboratoires publics ou privés. 


\section{A multi-agent knowledge model for SMEs mechatronic supply chains}

Jihene Tounsi

University of Savoie, SYMME-Polytech'Annecy

12, chemin de Bellevue BP 80439

74940, Annecy-le-Vieux

Jihene.tounsi@univ-savoie.fr

Georges Habchi

University of Savoie, SYMME-Polytech'Annecy

12, chemin de Bellevue BP 80439

74940, Annecy-le-Vieux

Georges.Habchi@univ-savoie.fr

Julien Boissière

University of Savoie, LISTIC-Polytech'Annecy

12, chemin de Bellevue BP 80439

74940, Annecy-le-Vieux

Julien.Boissiere@univ-savoie.fr

Selma Azaiez

CEA, LIST (Embedded Real-Time System Foundations Laboratory)

Mail Box 94 - F91191 Gif sur Yvette Cedex

Selma.azaiez@cea.fr

Abstract: The main concern of this research work is to analyse and model supply chains (SCs) in the particular context of small and medium enterprises (SMEs) in the field of mechatronic. The study is based on the analysis of the organisational features, the actors' behaviour, and performance considerations. The development of the model relies on an iterative framework that progressively integrates different aspects into the model. This framework is the ArchMDE process, which is based on MDE (Model Driven Engineering). A major feature of this work lies in its contribution to two different areas of research. The first contribution of the work is to propose a generic metamodel for SCs. Based on a literature review, an incremental framework is proposed for the modelling of SCs in terms of concepts, structure and relationships. The application of the framework to the studied context is described and its result is a domain-metamodel for SCs. The second contribution of this work lies in the formalisation of the dynamic behaviour of the concepts in the metamodel. This formalisation is based on the multi-agent approach. An agentification of the metamodel is thus drawn, thanks to the natural links between multiagent theory and SC reality. This step leads to an agentified-domain-metamodel which also includes the monitoring of the SC and synchronisation protocols. By adding relationships and dynamic behavior aspects, we obtain a 
metamodel of the domain that can be implemented, with its static and dynamic aspects. To validate this model, an industrial case study is detailed and has been instantiated and encoded in JAVA.

Keywords: SME, supply chain, modeling, multiagent

\section{Introduction}

The supply chain concept was born in the 90's when management techniques in the business world were evolving from separated to collaborated logistics. It is well known that the supply chain is a complex macro system. This complexity is firstly due to the variety of the involved organizations and to the diversity of relationships between them, and secondly it results from the decisionmaking mechanism between these companies. Thus, the success and subsistence of a company in the economic market lie in its ability to integrate managerial processes but also to coordinate with other actors (Drucker, 1998; Lambert and Cooper, 2000). In this context, Small and Medium Enterprises (SMEs) evolve in an unstable and complex network. In order to guarantee its role in a supply chain, a SME must be able to support the inherent requirements of the supply chain (lead times, consumer satisfaction, etc.) and the external requirements due to the environment (unpredictable mutation, competition, etc.). Consequently, SMEs have to collaborate together in order to achieve their goals without losing their autonomy and identity (Julien, 1997; Villarreal et al., 2005).

The industrial environment of the Savoie region in France is mostly composed of small and medium size manufacturers or subcontractors in the mechatronic industry. These SMEs are clustered into SME networks in order to achieve a common goal in a complex overall supply chain. Indeed, according to some field investigations, three major features of the supply chain which integrates SME clusters and especially mechatronic ones arose (Tounsi et al., 2008). Firstly, in this context, a supply chain is a complex system. This complexity is due to the number of autonomous actors and to the number of SME networks which work together to achieve a given process. Secondly, the studied SMEs are not necessarily located in the same geographical area as the other nodes of the supply chain. Finally, they face a lack of visibility in the overall supply chain as a result of the two previous characteristics. In fact, these sites only have local visibility but are coordinated with other sites through the flow of products. Due to the complexity, the decentralization and the lack of visibility in the overall supply chain, studying the structure and the behavior of the supply chain in the SMEs of mechatronic field has become a challenge and producers in the county of Savoie have expressed a growing need.

However, the study of and the experimentation with the overall supply chain integrating mechatronic SMEs cluster are difficult to implement on actual industrial systems. Thus, in order to facilitate the study and analysis of the network, it is necessary to propose a modeling solution which reflects the actual system and is able to simulate its behavior. In the light of this perspective, this paper proposes a knowledge-model based on a development process (ArchMDE) that aims to identify and model the domain concepts based on the multiagent system. Hence, the work described here is a combination of two research areas. The first one (industrial engineering scope) proposes a modeling approach using different layers that represent different views of the system 
(i.e. the system refers to a supply chain). The representation of the domain concepts within the models allows one to capitalize on the know-how and then facilitates the re-use of the supply chain concepts within different contexts. The second one (the computer engineering scope) outlines the transition from the identification step of the domain structure to the study step for the dynamics behavior of the domain concepts. This study is based on combining the domain concepts and the multiagent ones.

In this paper, we highlight the research work through these two fields. Hence, the article is organized into six major sections. The first presents existing supply chain modeling approaches and focuses on the multiagent modeling one. In addition, motivations for the agent paradigm to model the supply chain are clarified. In the second section, the ArchMDE (Architecture Model Driven Engineering) development process is introduced and its contribution to this research is detailed. Then, the third and fourth sections present the different steps to generate the conceptual metamodel and its agentification according to the development process described. The fifth section integrates the dynamic behavior of the different agentified concepts based on multiagent theory. Finally, the last section highlights the transition from the modeling phase to the implementation one. This proposal ends with a conclusion and some perspectives for this research.

\section{Literature review}

\section{Supply chain modeling approach}

Beamon (Beamon, 1998) and Labarthe et al. (Labarthe et al., 2007) distinguish three main types of approach for supply chain modeling: organizational, analytical and simulation.

The organizational approaches rely on process modeling based on the systems theory. This modeling approach is subdivided into two research scopes: hierarchical or heterarchical one. Berger et al. (Berger et al., 2010) explain the difference between these two research scopes. However, the supply chain models generated using these approaches are usually unable to evaluate the dynamic behavior of the system over time when facing stochastic environmental stimuli.

The analytical approaches rely on mathematical formalizations of the chain. The models obtained are simplified, usually require restrictive assumptions, and are limited in taking time into account. Two such approaches are the control theory approach, based on differential equations, and the operational research approach, which relies on optimization theories. Parunak et al. (Parunak et al., 1999) and Sarimveis et al. (Sarimveis et al., 2008) provide a review about the application of the analytical approaches for modeling the supply chain management.

Supply chain modeling and simulation (M\&S) is based on system dynamics and on the behavior of different autonomous entities. It is subdivided into two different scientific research topics: continuous simulation and discrete event simulation. Currently discrete event simulation is the mainstream approach (Terzi and Cavalieri, 2004).

In this research work, the M\&S is suitable to study the structure and the dynamic behavior of the SMEs mechatronic supply chain. In fact, modeling is a mechanism that reflects the actual system and provides a very powerful decision-making tool when coupled with simulation. The literature is 
unanimous on the positive role of M\&S in the study, analysis and performance evaluation of complex systems. For example, some authors (Ingalls, 1998; Lee et al., 2002; Longo and Mirabelli, 2008) highlight the features and advantages of a decision-making tool based on modeling and discrete event simulation.

The M\&S approach was adopted in several works in order to reduce the complexity of the supply chain and evaluate its performance (Bagchi et al., 1998; Labarthe et al., 2007). M\&S translates the supply chain conceptual model and recreates the complexity and highly stochastic environment of a supply chain. The conceptual model defines concepts (involved entities) and parameters that give a supply chain manager the possibility to analyze different scenarios by changing the input parameters (Longo and Mirabelli, 2008).

In supply chain M\&S there are two main types of modeling: equation-based modeling and agentbased modeling. Parunak et al. (Parunak et al., 1998), Janssen (Janssen, 2005) and Monteiro et al. (Monteiro et al., 2008) have shown that multiagent systems and agents are more suitable for modeling the dynamics behavior of the complex network manufacturing system and to study the impact of flow coordination between different entities than equation-based modeling.

In this work we have chosen to adopt the multiagent system to model and simulate the supply chain in the SME context. The motivations for this choice are highlighted in the next subsection.

\section{Agent-based modeling for supply chain}

The Multiagent system is a recent M\&S paradigm of complex systems. The Multiagent approach was born by combining two research fields: "artificial intelligence" and "object-oriented modeling”. Demazeau (Demazeau, 1996) defined the multiagent system as a set of four main views named the "Vowel approach" also known as the "AEIO approach":

- Agent view (A): describes the internal structure of an agent. An agent is a computer system able to act autonomously in a given environment in order to meet the design objectives of the model (Wooldridge, 2002). The scientific community distinguishes three kinds of agent according to their decision-making model and degree of intelligence; (i) the reactive agents (Brooks, 1991); (ii) the cognitive agents (Wooldridge, 1999; Bratman et al., 1998); (iii) the hybrid agent (Fischer et al., 1995).

- Environment view (E): describes the external environment in which an agent evolves.

- Interaction view (I): describes the dynamic relationships between agents through protocols or interaction language.

- Organization view $(O)$ : describes the structure of the whole system in terms of agent groups, hierarchy, relationship and the structure of the other entities, which constitute the environment.

The AEIO approach breaks the whole multiagent system down into several modules. This modularity facilitates the reuse of the different modules according to requirements. In addition to this modularity, agents are more suitable for applications that are decentralized, changeable, illstructured (dynamic structure) and complex (Parunak, 1998). Also, Janssen (Janssen, 2005) highlights the main characteristics that make multiagent system privileged to model the dynamic behavior of supply chain. So, the multiagent approach provides a framework that is naturally 
oriented to model the supply chain. By comparing the supply chain and the multiagent system characteristics, similar concepts and the same organizational practices arise. Both are composed of autonomous actors or entities which evolve in an organization and interact to achieve a collective purpose. This analogy, which is described in more details in (Yuan et al., 2002) leads to the multiagent approach being a privileged way to model supply chain systems.

Many researchers apply multi-agent system to carry out their scientific projects since the 90s. In literature, several works draw up an overview of researches based on multiagent technology to understand and model the supply chain. Among these works, we can cite in the 90s (Parunak, 1999; Shen and Norrie, 1999) and more recently (Fung and Chen, 2005; Shen et al., 2006; Monostori et al. 2006; Labarthe et al., 2007; Frayret et al., 2008; Al-Mutawah et al., 2009; Oztemel and Tekez, 2009). However, most multiagent works deal with issues of one enterprise point of view. This is a lack for supply chain researches in the SME clusters integrating the overall supply chain.

\section{The ArchMDE development process}

In this paper, the goal is to combine multiagent concepts and supply chain ones in order to build an agentified conceptual model for supply chain in SMEs context. To reach this purpose, the modeling approach recently proposed within a $\mathrm{PhD}$ research work (Azaiez, 2007) is used. This approach is based on Model-Driven Engineering (MDE) (Kent, 2002) which founds its developing process on producing several interrelated models. MDE promotes the separation and combination of concerns in software engineering. Applying this approach allows one to control the software development process during its different phases (from analysis to implementation).

One of the most important issues of the MDE approach is the metamodelling one. A metamodel targets important aspects of software. It defines the domain concepts, their relationships and their properties. In the MDE approach, the metamodels are not only descriptive models. They are the core of the development. All models produced in the different development phases (from analysis through implementation) have to conform to the metamodel.

In the ArchMDE approach, two types of metamodels are identified: a domain metamodel that describes functional concepts and properties related to a particular domain (i.e. a SME supply chain) and a computer modeling metamodel (i.e. a multiagent system). A combination of both metamodels will generate an agentified metamodel, that constitutes the starting point of the conceptual models. From this last metamodel, different functional models are described in order to introduce the functionalities of the system (Fig 1). Finally, the use of a platform metamodel is necessary to generate the program code. 


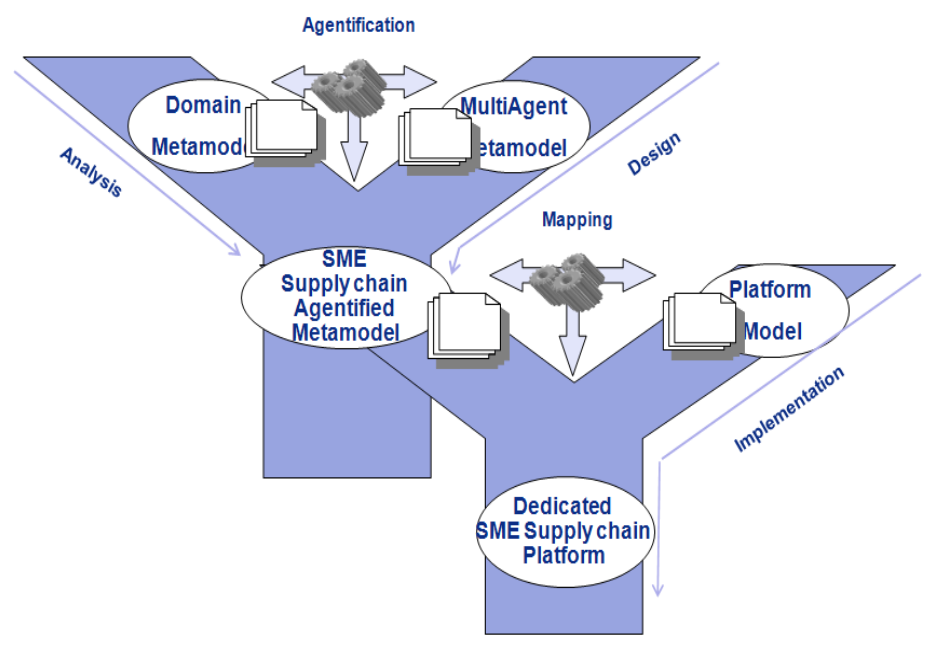

Fig 1. ArchMDE development process

This approach is of great interest to fill in the existing gap between the design and the implementation phases. The following sections describe the different steps of the ArchMDE process:

- The conceptual step defines the domain metamodel (section 4).

- The agentification process is introduced through the multiagent metamodel and the analogy between the multiagent concepts and the domain ones (section 5). The agentification is achieved by integrating the dynamic behavior into the agentified SME supply chain metamodel (section 6).

- The implementation phase focuses on the transition from the modeling step to the encoding one.

\section{Conceptual domain metamodel}

According to the ArchMDE development process, the first modeling step involves the definition of the domain conceptual model. This step leads to the identification of the main concepts of SME mechatronic supply chains. To achieve this objective, we follow a methodology based on existing conceptual modeling visions in the literature (Tounsi et al., 2008). In this methodology, the visions are organized into three steps. Each step addresses concepts related to supply chains. These concepts and their relationships will then be gathered within a domain metamodel that will be expressed using UML (a semiformal Unified Modeling Language). The following section presents this methodology.

\section{Conceptual modeling methodology}

To identify the properties and concepts of the supply chain domain, an incremental methodology combining three visions is proposed: product vision, structure vision and process vision. In each step, a vision is applied to build or to refine the conceptual model. The result of each step (intermediary model) is the input of the next one. Therefore, at the end of the 3 steps, a final architecture of the conceptual model is generated (Fig 2). 


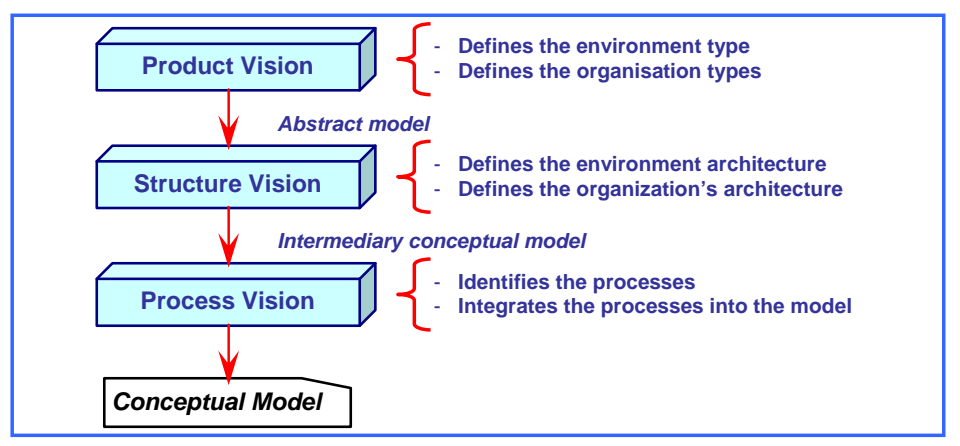

Fig 2. Conceptual Modeling Methodology Framework (Tounsi et al., 2008)

\section{Step 1: Product Vision}

This vision considers the supply chain dedicated to a particular product (or a family of products) from the raw materials through the final goods. It focuses on the product flow to define the environment and organizations involved in its management (Thierry, 2003). In the methodology framework, the Product Vision leads to the construction of a first abstract model of the supply chain involving the environment and organizations:

- The environment is characterized by the physical flows and the different steps of the product transformation as well as the related disturbances.

- The organizations are the entities carrying out one or several product transformation stages and the physical flow management. In the studied context, the supply chain is essentially made up of SMEs. The organizations involved can be a network of firms that collaborate to accomplish one or several transformation stages.

\section{Step 2: Structure Vision}

This vision has been proposed by Cooper et al. (Cooper et al., 1997). It considers the architecture of the supply chain made up of: actors (decision-making actors and synchronization actors), network structure (roles in the network and the number of actors for each role) and relationship characteristics between actors. So, on the basis of the abstract model provided by the previous step, the Structure Vision details the organizations involved and the physical environment:

- The environment is the part containing the physical flow. Therefore, the product flow and the resources used to achieve its transformation have to be described.

- The organization consists in identifying and prioritizing the actors in the network according to their involvement in the different levels of decision-making as well as the tasks that will be assigned. The information flow management depends on the decisionmaking level.

At this step, a more detailed intermediate model is built.

\section{Step 3: Process Vision}

This vision is based on the process classification according to the decision-making level (Stevens, 1989; Chopra and Meindl, 2001): strategic, tactical and operational. 
While applying Process Vision, the various categories of processes are identified and integrated into the previous intermediate model. This can be done according to the decision level but it also depends on the relationships between the actors. These relationships can be classified into two categories:

- Synchronization: contains processes for exchanging information and physical flows according to a process program developed and predefined by the decision-making layers.

- Management and control: contain processes that ensure suitable decision implementation in the perspective of a continuous improvement of processes in terms of added value.

This step leads to a refined conceptual model of the supply chain.

\section{Domain model concepts}

This section presents the concepts that constitute the domain model. By applying the methodology, several concepts, processes and the architecture of the model were identified. Based on these concepts, a metamodel of supply chain is proposed.

\section{Step 1: Applying Product Vision}

By applying the Product Vision, a first abstract model of the supply chain is built. It is composed of (Fig 3):

- Environment: the space allocated to the product flow and management through the internal resources as well as the external elements able to influence supply chain activities.

- Sub Supply Chain (SSC): represents a group of SMEs which collaborate to achieve an internal aim and/or the overall objective of the supply chain. The SSC is responsible for the management of the product flow in a certain stage of its life cycle.

- Perimeter of influence: represents the visible part of the environment to the SSC on which it can act by internal conferring (if the action does not disturb the environment located outside its visibility) or by conferring with another SSC.

- $\quad$ Shared perimeter of influence: represents the area of the flow transfer between two SSCs. It is a shared zone where SSC coordinates their activities to allow the flow transfer.

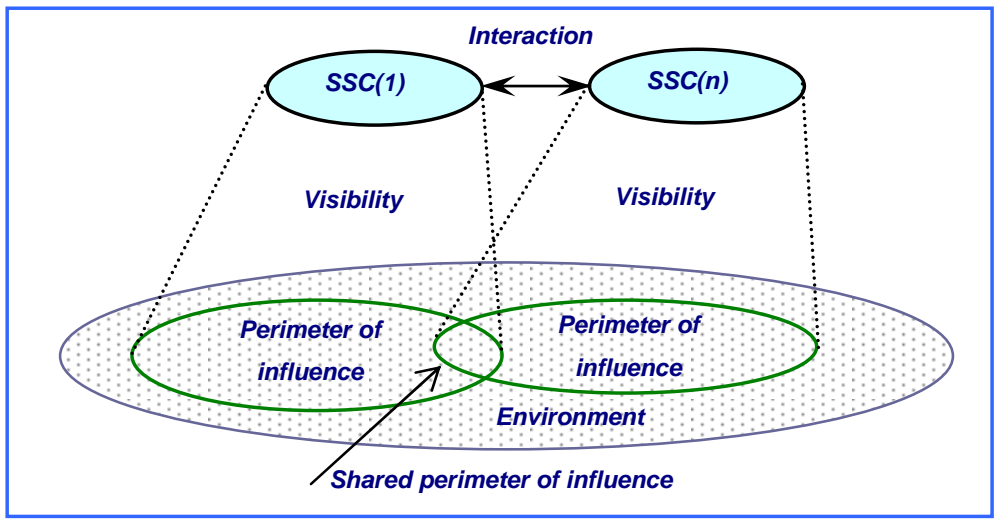

Fig 3. The Abstract Model (Tounsi et al., 2008) 
Fig 4 shows the domain metamodel which reflects this conceptual abstract model using UML language.

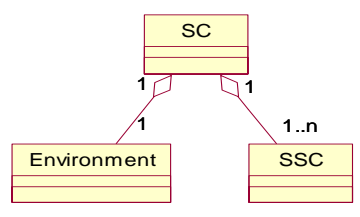

Fig 4. Abstract Domain Metamodel

\section{Step 2: Applying Structure Vision}

By applying Structure Vision, the previous abstract model is refined. The internal architecture of the SSC and the visible part of the environment (the perimeter of influence) are described. As showed in Fig 5, the SSC model and its environment are based on three layers representing the different decision-making levels.

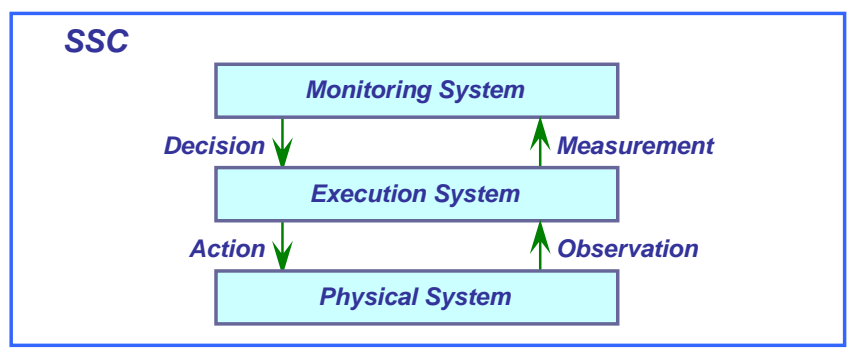

Fig 5. Layers of the SSC (Tounsi et al., 2008)

Each layer involves particular concepts and plays a specific role in the SSC:

- The Monitoring System is the intelligent layer of the SSC. It controls and monitors the two other layers through the information provided by the Execution System. Monitoring Actors (MA) modeling the intelligent actors of SSC are the main elements of this layer. They establish metrics to evaluate the performance of the group and consequently act on the other two layers. Hence, MAs are the components responsible of controlling and decision-making into a SSC and of the coordination of the activities for the overall supply chain.

- The Execution System is the reactive layer of the SSC. It has two main roles: (i) it ensures the synchronization of the physical flow according to the information gathered from the Physical System, (ii) it observes and corrects the Physical System if a disturbance occurs. In abnormal situations, the Execution System refers to the Monitoring System for coordination and decision-making. Executive Actors (EAs) are the main entities of this layer. EA mainly models the reactive actor in the Execution System. However, occasionally MA can appear in this layer with reactive behavior.

- The Physical System is the visible part of the SSC environment. It corresponds to the SSC's perimeter of influence. This layer is composed of non-decisional elements controlled by the other two layers of the SSC. Two main concepts are identified: the Moving Entity (ME) modeling the product in circulation and the Resource modeling production means. 
Fig 6 shows the first conceptual abstract model refined in a domain metamodel. In one hand, we have integrated the identified concepts of each layer. In the other hand, an abstract class “Actor” is added to the metamodel for implementation purposes. In fact, the “Actor" class defines the structural characteristics and behavior of a decisional entity. So, both the EA and the MA inherit from this class. However, the "EA" class defines the specific characteristics of an executive actor and likewise for "MA" class.

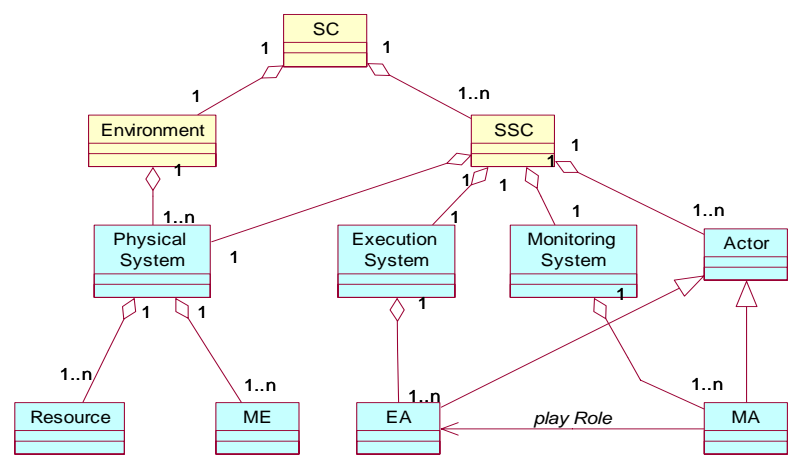

Fig 6. Intermediary Domain Metamodel

\section{Step 3: Applying Process Vision}

The object of the last step is to identify and integrate the different kinds of processes into the model. Table 1 gives a classification of the processes identified according to their role in decisionmaking. In the Physical System, the Physical Processes (PhPs) have been identified. A PhP describes the sequence of the processing stages of a product. It is a concept to be integrated within a domain metamodel in order to define the tasks that can be handled by the Execution System.

Table 1. Process Classification

\begin{tabular}{|l|l|l|}
\hline SSC Layer & Process Family & Role \\
\hline \multirow{3}{*}{$\begin{array}{l}\text { Monitoring } \\
\text { System }\end{array}$} & Strategic Processes (SPs) & - Coordinate long term decisions \\
\cline { 2 - 3 } & $\begin{array}{l}\text { Monitoring and Control } \\
\text { Processes (MCPs) }\end{array}$ & $\begin{array}{l}\text { - Monitor SSC activities } \\
\text { - Drive and evaluate SSC performance in the overall } \\
\text { supply chain }\end{array}$ \\
\hline $\begin{array}{l}\text { Execution } \\
\text { System }\end{array}$ & $\begin{array}{l}\text { Operational Control } \\
\text { Processes (OCPs) }\end{array}$ & - Synchronize and control the physical system \\
\hline $\begin{array}{l}\text { Physical } \\
\text { System }\end{array}$ & Physical Processes (PhPs) & - Define the transformation routings of products \\
\hline
\end{tabular}

The processes identified in both Monitoring and Execution Systems are management processes. Hence, they represent the dynamic behavior of the SSC. This behavior is induced by control and monitoring decisions that come from either the SSC or the overall supply chain. It uses a communication mechanism (coordination, collaboration or cooperation).

In order to model management processes and communication mechanisms, more informational elements are needed for EAs and MAs to ensure their role in the domain model. Thus, decisional actors of the SSC (EA and MA) need three conceptual elements that consolidate their internal architecture:

- Indicator: is used by actors for two different tasks. In fact, the EA actors control and detect Physical System deviation by comparing the value of an indicator with its fixed 
objective. As for MA actors, they evaluate the internal performance of the SSC but also in the overall supply chain.

- Action: Actors apply actions when facing indicator deviation.

- Organizational Knowledge: is an actor's database that stores information about his acquaintances. For example, if an actor " $\mathrm{A}$ ” is an acquaintance of an actor " $\mathrm{B}$ ” this means that " $\mathrm{B}$ " owns information about the identity, the behavior, the capabilities and the resources of the actor "A". Reciprocally, the actor "A" owns the same information about the actor "B". According to this, each actor (EA and MA) owns knowledge about resources of all actors in the same SSC. However, the MA actor involves in the overall supply chain. So in addition to internal acquaintances, the MA owns limited knowledge about the others MAs of the overall supply chain. This knowledge requires continuous updating.

In the same way, the intelligent behavior of the MA requires the definition of other conceptual components:

- Objective: models the strategic goal of the SSC. According to this aim, the SSC coordinates its activities with other SSCs in the overall supply chain.

- Constraint: is a knowledge that an actor must consider to reach the goal of the overall supply chain or the SSC's one.

Through the Process Vision, the previous metamodel and its concepts are refined by integrating identified concepts. Figure 7 presents an UML representation of the final domain metamodel for the supply chain in SMEs context. It corresponds to the final conceptual model with its associated concepts regardless of computer technologies.

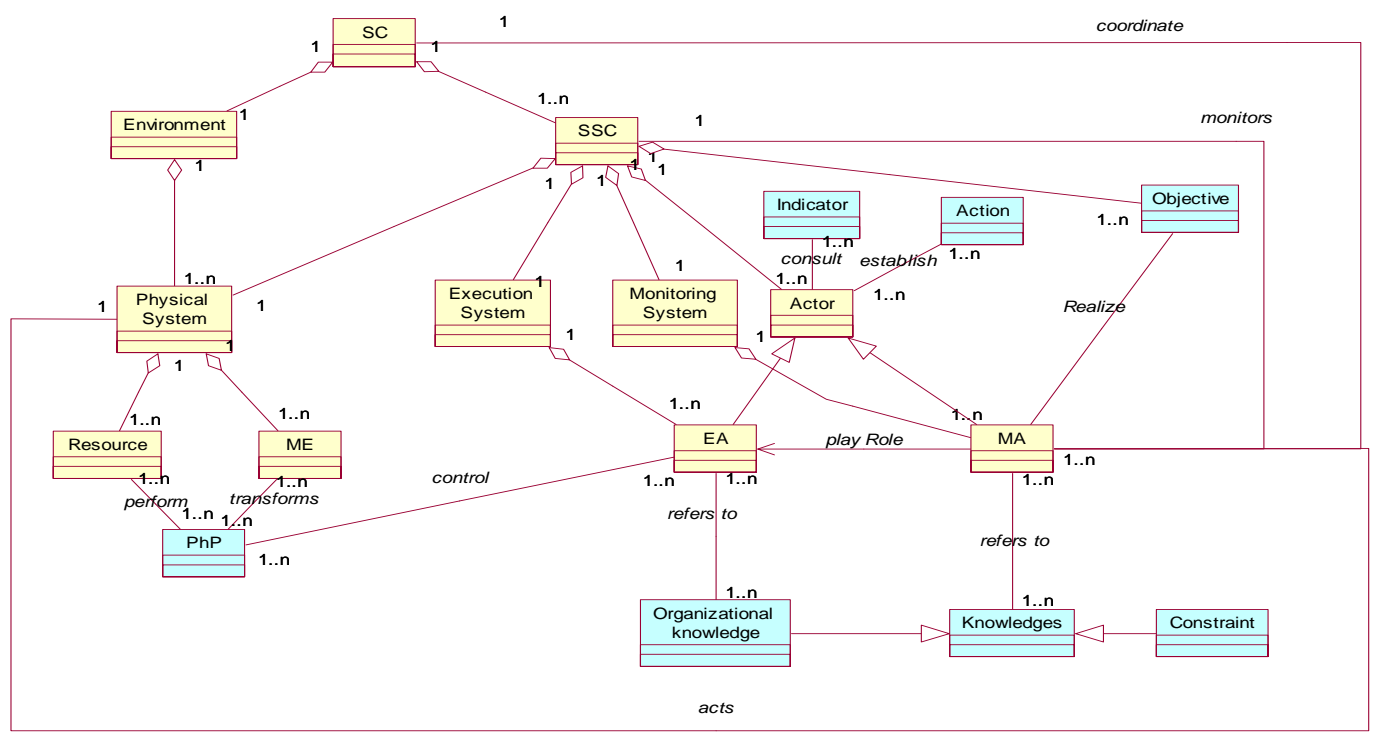

Fig 7. Final Domain Metamodel

\section{Agentified SMEs supply chain metamodel}

In this section, the domain metamodel is merged with an agent metamodel using the ArchMDE methodology (Azaiez, 2007). Thus, this section is divided into two parts: the first one outlines the 
properties of each multiagent concept according to the "AIEO approach" and the second part highlights the agentification of the domain metamodel.

\section{Multiagent metamodel}

The "AIEO approach" breaks the whole multiagent system down into four views: agent view, environment view, interaction view and organization view. Figure 8 shows multiagent concepts according to each view and the links between them.

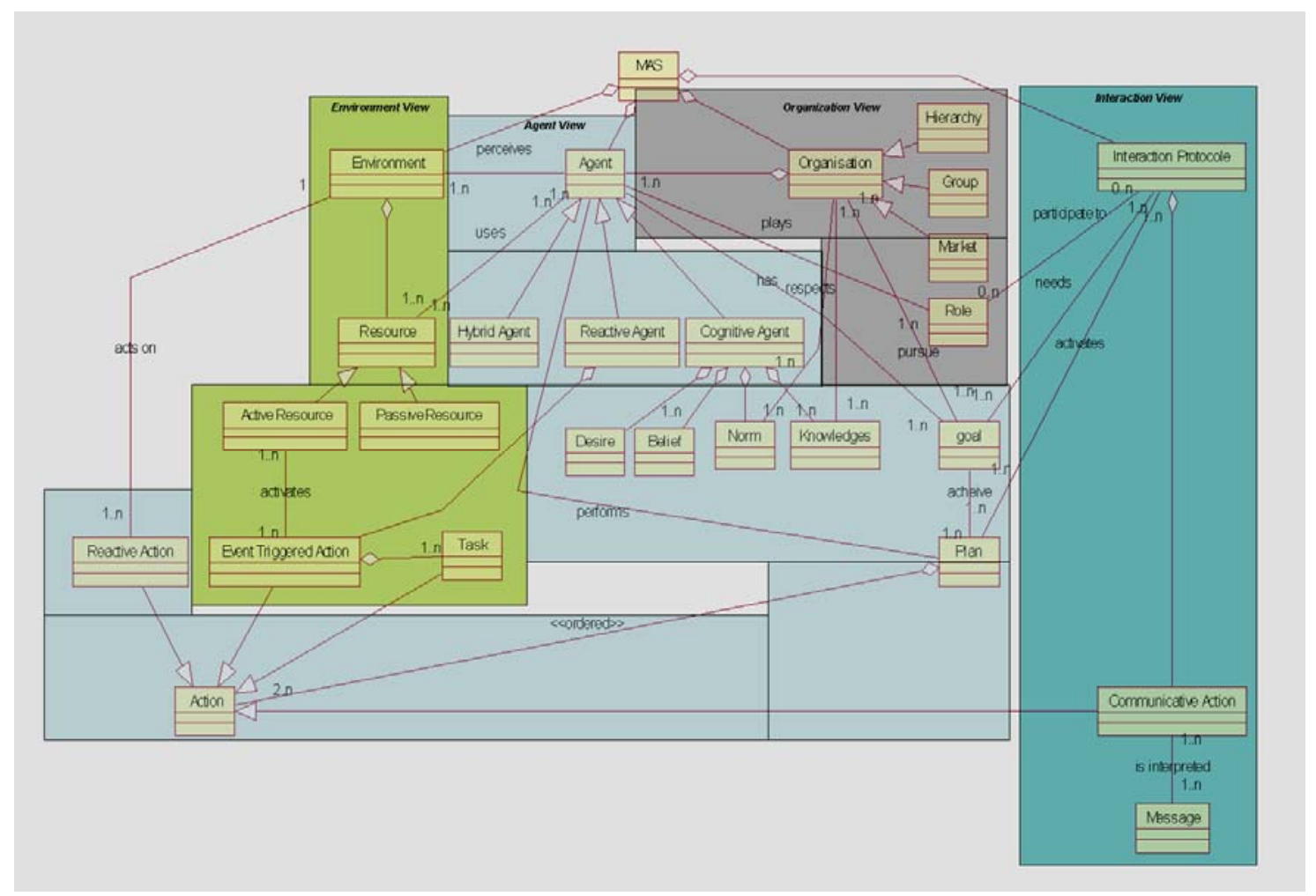

Fig 8. Agent Metamodel (Azaiez, 2007)

The "agent view" defines the agent metamodel composed of the following concepts:

- "Agent” identifies different kinds of agent according to the decision-making capacity of the agent (reactive agent, cognitive agent and hybrid agent).

- "Cognitive agent” defines an agent with cognitive abilities. The metamodel highlights the main concepts modeling the BDI agent (Belief, Desire, Intention=plan).

- "Reactive agent” defines an agent with reactive abilities.

- "Hybrid agent” defines an agent with hybrid intelligence (cognitive and reactive abilities).

- "Goal" defines the aim that agent should achieve.

- "Knowledge" and "norm” define all the knowledge and norm necessary for the agent to achieve his goal.

- "Plan” represents an action plan implemented by the agent. The plan is composed of one or several elementary actions.

- "Reactive action" is an action implemented by the reactive agent. 
The "environment view" focuses on all the elements external to the agent that allow him to reach his goal or activate his behavior through events. The elements belonging to the "environment metamodel” are the following:

- "Active resource" represents the resources that activate the behavior of the agent by generating events or triggers.

- "Passive resource" defines the resources the agent needs to accomplish his task.

- “Event triggered action” represents event that resources activate. An event is composed of one or more tasks.

The "interaction view" describes the dynamic relations between the agents. This interaction is a structured exchange of messages between the agents through a specific protocol or language. So the "interaction metamodel” highlights the following concepts:

- “Interaction protocol” represents the interaction protocol adopted by the agents.

- “Communicative action” represents an elementary action of communication that is part of the "interaction protocol”.

- "Message" is a set of information exchanged between the agents through the "interaction protocol”. The agent interprets the message based on the communicative action.

Finally, the "organization view" describes the structure of the whole system. The "organization metamodel” is made up of the following concepts:

- “Organization” defines the system topology (hierarchy, group or market).

- "Role" represents different roles that the agent could play.

\section{Agentification of the domain metamodel}

This step of the ArchMDE methodology consists in merging the multiagent metamodel with the domain metamodel. Hence, on the one hand, a metamodel defines a multiagent system according to the "vowel approach" (Fig 8). On the other hand, a domain metamodel describes the supply chain in SMEs mechatronic context (Fig 7). A correspondence between the multiagent concepts and those of the domain is then carried out according to their properties and their roles in the metamodel. Table 2 summarizes the correspondence between these concepts in order to achieve the agentified metamodel for SME mechatronic supply chains.

Table 2 Correspondence between the domain and multiagent concepts

\begin{tabular}{|l|l|l|}
\hline Domain concepts & $\begin{array}{l}\text { Multiagent } \\
\text { concepts }\end{array}$ & Description \\
\hline $\begin{array}{l}\text { Supply Chain } \\
\text { (SC) }\end{array}$ & MAS & $\begin{array}{l}\text { By analogy, the root of the domain metamodel corresponds } \\
\text { to the root of the multiagent system. }\end{array}$ \\
\hline Environment & Environment & $\begin{array}{l}\text { In both metamodels, the environment is the physical space } \\
\text { defining all things that are external to the agents and } \\
\text { necessary in order to manage the SC. }\end{array}$ \\
\hline $\begin{array}{l}\text { Sub Supply } \\
\text { Chain (SSC) }\end{array}$ & Organization & It is an organization made up of two groups of agents. \\
\hline Physical System & Resource & $\begin{array}{l}\text { It is all the resources needed for one agent or a group of } \\
\text { agents to manage the group (perimeter of influence). }\end{array}$ \\
\hline Resource & Passive Resource & It is a resource allocated to the agent to perform its task. \\
\hline $\begin{array}{l}\text { Moving Entity } \\
\text { (ME) }\end{array}$ & Active Resource & $\begin{array}{l}\text { The ME represents the product in circulation. It activates } \\
\text { the behavior of the reactive agents. }\end{array}$ \\
\hline Physical Process & Task & It is a task or a physical activity to be handled by reactive \\
\hline
\end{tabular}




\begin{tabular}{|c|c|c|}
\hline$(\mathrm{PhP})$ & & agents. \\
\hline $\begin{array}{l}\text { Monitoring } \\
\text { System }\end{array}$ & Group & $\begin{array}{l}\text { It is a group of cognitive agents which collaborate in the } \\
\text { SSC and coordinate the activity of the organization with } \\
\text { other organizations. }\end{array}$ \\
\hline $\begin{array}{l}\text { Execution } \\
\text { System }\end{array}$ & Group & $\begin{array}{l}\text { It is a group of reactive agents which collaborate in the } \\
\text { SSC. }\end{array}$ \\
\hline Actor & Agent & $\begin{array}{l}\text { An actor can be a cognitive agent or a reactive agent } \\
\text { according to its decisional characteristics. }\end{array}$ \\
\hline $\begin{array}{l}\text { Executive Actor } \\
\text { (EA) }\end{array}$ & Reactive Agent & $\begin{array}{l}\text { EA perceives the physical system and acts on it according } \\
\text { to the observation. }\end{array}$ \\
\hline $\begin{array}{l}\text { Monitoring Actor } \\
\text { (MA) }\end{array}$ & Cognitive Agent & $\begin{array}{l}\text { According to the collected information and the history of } \\
\text { the situation and action, the group of MAs monitors the } \\
\text { SSC to reach a goal and accomplish its activity. }\end{array}$ \\
\hline Objective & $\begin{array}{l}\text { Goal } \\
\text { Desire }\end{array}$ & $\begin{array}{l}\text { A SSC has a goal to reach. This goal is coordinated with } \\
\text { other nodes' goals. In addition, each MA has a personal aim } \\
\text { for each indicator. This kind of "Objective" is modeled by } \\
\text { the "Desire" of the BDI agent (MA). }\end{array}$ \\
\hline Indicator & $\begin{array}{l}\text { Belief } \\
\text { Perception }\end{array}$ & $\begin{array}{l}\text { The agents act on the environment according to the } \\
\text { indicator measures. In this case, an indicator is modeled by } \\
\text { the "Perception" of an agent. However, a MA monitors the } \\
\text { SSC according to the history of these measures. So, an } \\
\text { "Indicator" is modeled by the "Beliefs" of BDI agent. }\end{array}$ \\
\hline Action & Plan & $\begin{array}{l}\text { It is an action or a set of actions to apply when facing a } \\
\text { disturbance. }\end{array}$ \\
\hline Knowledge & Knowledge & $\begin{array}{l}\text { It is all the knowledge needed by the agents to act in an } \\
\text { appropriate way. }\end{array}$ \\
\hline $\begin{array}{l}\text { Organizational } \\
\text { knowledge }\end{array}$ & Knowledge & $\begin{array}{l}\text { Each agent has a list containing the information about other } \\
\text { agents from the same SSC or the overall SC. This list stores } \\
\text { knowledge about the name of the agent, the task that it } \\
\text { performs and its resources. }\end{array}$ \\
\hline Constraint & Knowledge & $\begin{array}{l}\text { The MAs make decisions according to their objectives and } \\
\text { their beliefs. At the same time, there are some constraints } \\
\text { (about product or other SCs where the group is involved) } \\
\text { that the group of MAs must take into account when making } \\
\text { decisions. }\end{array}$ \\
\hline
\end{tabular}

After the agentification process, we obtain an agentified domain metamodel as presented in Figure 9. The domain metamodel and the multiagent one are separated for more clarity. 




Fig 9. The agentified SME supply chain metamodel

\section{The integration of processes into the metamodel}

So far, the static part of the domain metamodel has been created. In this part, we define the dynamic behavior of the concepts based on the multiagent tools and theory. Indeed, this dynamic is described by the implementation of interaction protocols according to the "process vision" and the communication mechanisms.

Firstly, the "process vision" allow us to define two scenarios: (1) the synchronization of the physical processes and (2) the monitoring and the control of processes.

Secondly, a communication mechanism is "a framework formalizing interaction between different actors in the network according to their managerial relationship characteristics" (Tounsi et al., 2010). The study of the domain identifies two kinds of communication framework. In fact, in the overall supply chain, SSCs coordinate their activities in order to achieve the common objective of the overall supply chain. Within the SSC, the actors collaborate to achieve a local goal.

This section describes the different protocols implemented in the agentified domain metamodel taking into account the different scenarios of the "process vision" and the communication mechanisms.

\section{Synchronization of the physical processes}

The SSC is responsible for the synchronization of the Physical System involved to achieve its task. This activity consists in applying a communication protocol relative to the nature of the interaction framework. In this section, the collaboration and coordination processes are described in order to 
be implemented in the Execution System and Monitoring System and to synchronize the Physical System.

\section{Integration into the "Execution System"}

In accordance with the agentified domain metamodel, the Execution System is responsible for the synchronization of the physical process $(\mathrm{PhP})$ in common situations. Indeed, Executive Actors (EAs) which are reactive agents, synchronize $\mathrm{PhP}$ by taking into account the availability of resources.

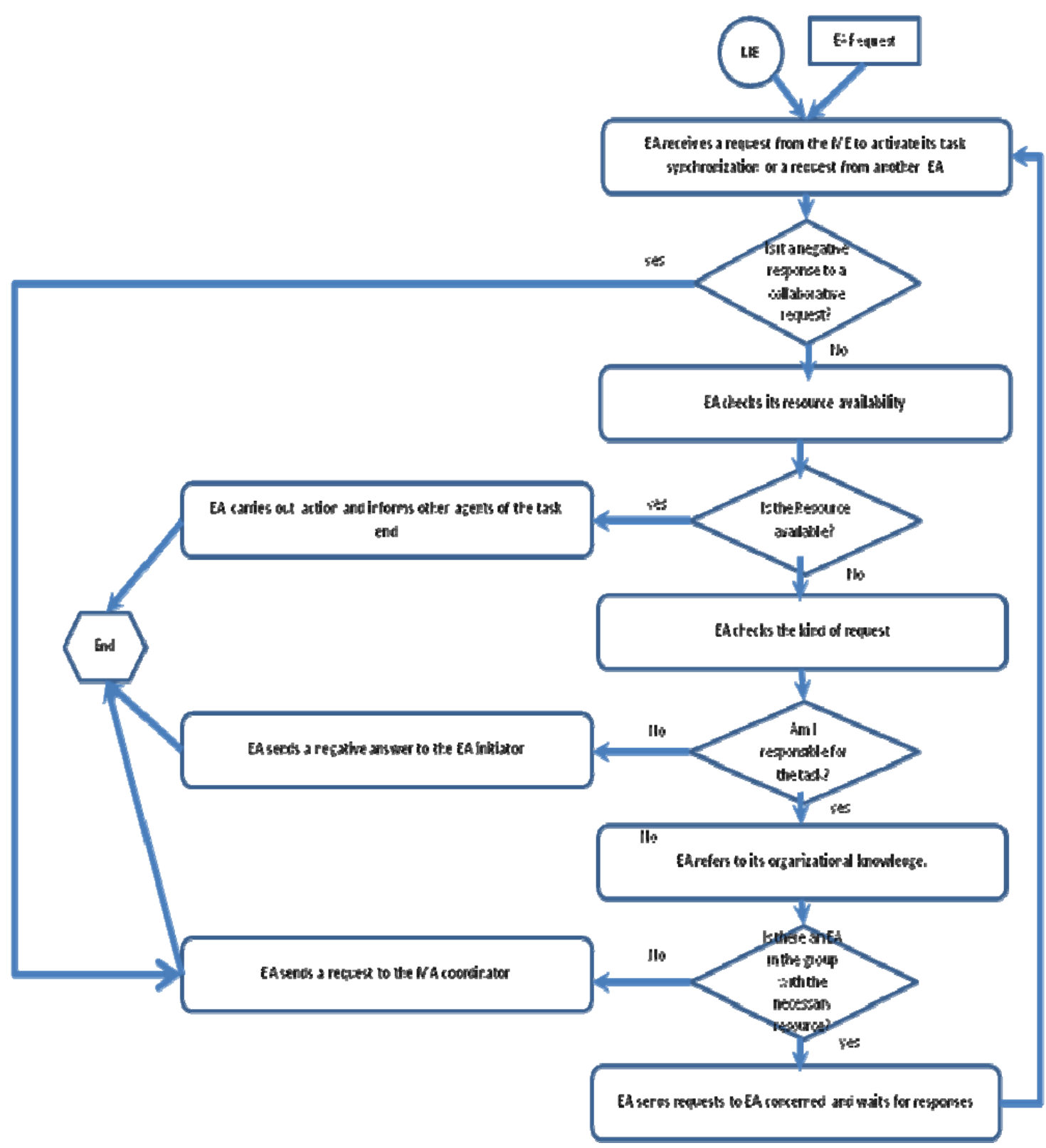

Fig 10. EA Synchronization behavior (Tounsi et al. 2009a)

Figure 10 describes the Executive Actor's (EA) behavior in its role of synchronizing the physical flow (ME). An EA receives a request and reacts according to its type. Three types of request can be distinguished: (1) a ME request, (2) a collaborative request from another EA or (3) a negative response to a collaborative action initiated by the agent itself. The following sequence highlights the EA's behavior: 
- If the request is a negative response for the collaborative demand that the EA initiated, the EA sends a request to the coordinator agent (MA coordinator) of the monitoring system.

- If the request is a synchronization need coming from the Moving Entity (ME) or a collaborative request coming from another initiator agent, the EA checks the availability of the resources concerned.

- If the resource is available, the EA carries out its task, updates the state of the ME and informs the other agents within the executive system and the coordinator agent at the end of the action.

- If the resource is unavailable and the EA has been solicited by another executive agent to achieve the task, it sends a failure request to the initiator.

- If the resource is unavailable and the EA is in charge of the task, then it seeks within its organizational knowledge an agent from the Execution System of the SSC that might have the necessary resource.

- If the agent finds another agent within its organizational knowledge that can handle the task, it delegates the responsibility of the task. In this case, the collaboration process of the agent concerned will be activated and follows the same sequence.

- If the agent does not find another agent who has the resource necessary to handle the task, it sends a request to the coordinator agent. This agent is a monitoring agent (MA) that receives requests from the Execution System. The MA sends the information to other monitoring agents in the SSC in order to find a solution.

\section{Integration into the "Monitoring System"}

In unusual situations ${ }^{1}$, the Executive System refers to the Monitoring System. In this case, the group of MAs evaluates the situation according to the defined objective and establishes an action plan. If the objective is not reached, the MA needs to consult other SSCs to find a suitable solution. So, the agents adopt the protocol based on Contract Net Protocol to provide the coordination of the objectives. The synchronization protocol can be described according to the following steps:

- In the Monitoring System, a Monitoring Actor (MA) is responsible for checking all the requests received and sending them to other MAs in the layer. Three kinds of requests can be distinguished: (1) EA request, (2) reply to a help request or (3) a help request from another SSC in the overall supply chain.

- If a MA coordinator receives an EA request then it sends the information to other MAs. In this case, the group evaluates the situation according to the SSC's objectives. Two cases may arise: (1) the problem has no impact on satisfying the SSC's objectives or (2) the objective is deviated.

\footnotetext{
${ }^{1}$ Unusual situation is occurred when Executive System cannot propose a solution for a happened problem in the physical system.
} 
- If there is no impact on the objective, the MA tries to find an internal solution according to its desire, belief and constraints. If a solution can be found, the MA coordinator sends the actions plan to the Executive System.

- If the objective is deviated or an internal solution cannot be reached, the group of MAs sends a Help Request to other SSCs via the MA coordinator and waits for the responses.

- When all the replies have been received, the MA coordinator ranks them according to the reception date of the request. The list of responses will be sent throughout the Monitoring System. According to their beliefs, desires and constraints, the group of MAs chooses the most suitable answer and diffuses the action plan to the Execution System. In this case, the EA updates the state of the ME.

- If the request is a Help Request from another SSC, the MA coordinator sends the request to the Monitoring System. In this case, the Monitoring System evaluates the demands according to internal criteria (Objective, Constraint, Belief, and Desire). If the SSC can provide assistance, it makes an offer to the SSC initiator or it sends a negative response.

- The SSC initiator chooses the suitable offer and sends a confirmation to the selected SSC and a cancellation response to other bids.

The following figure shows the sequence of messages between the SSCs. This diagram represents the coordination process in the overall supply chain in order to synchronize the physical flow in the case of a disruptive case (SSC cannot reach the internal aim).

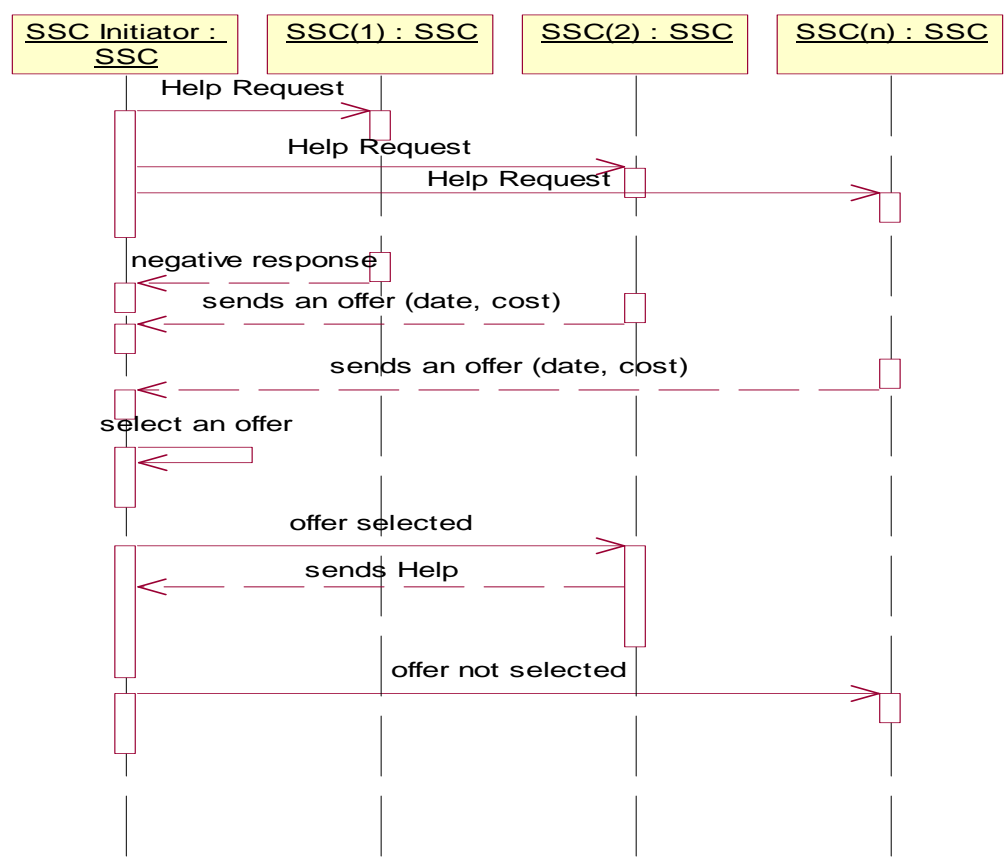

Figure 11. SSC coordination process (Tounsi et al., 2009b)

\section{Monitoring and control protocol}

This protocol describes the conditional preventive monitoring and control (based on measurements) and the corrective one (in case of disturbance) in the SSC or in the overall supply 
chain. The monitoring and control protocol is based on performance evaluation in both layers of the SSC: the Execution System and the Monitoring System.

At the end of the synchronization protocol, each actor updates the indicator measurement by evaluating its activity and related resources. In addition, the "Monitoring System” evaluates the local activity of the SSC and participates in the improvement of the performance of the overall supply chain. So, the following sequence describes the "Monitoring and control protocol" in the SSC's layers:

- At the end of its synchronization task, the EA evaluates the performance of its activity and related resources (the allocated space of the environment to the EA).

- According to this perception, the EA refers to the indicator base in order to detect a disturbance.

- If the EA finds a deviation, it seeks the cause of the disturbance.

- If the deviation is a common situation, the EA selects the appropriate action plan to solve the problem and applies it to the environment. After that, it sends measurement (or perception) to the MA coordinator which, in turn, sends the information throughout the Monitoring System. Then, each MA updates its belief.

- If a new situation occurs, the EA sends a failure control message to the MA coordinator. Then, the MA sends the information throughout the "Monitoring System" and each MA updates its belief.

- In this case, the "Monitoring System” analyzes the situation according to internal criteria (beliefs, desires, objectives and constraints).

- If the problem needs corrective maintenance, the "Monitoring System" generates an action plan and forwards it to the "Execution System". The actors of the SSC update their bases of actions.

- If the disruption does not affect the SSC, the "Monitoring System" applies a preventive action plan to avoid future disturbance.

Figure 12 shows the protocol for monitoring and control through an UML sequence diagram: 


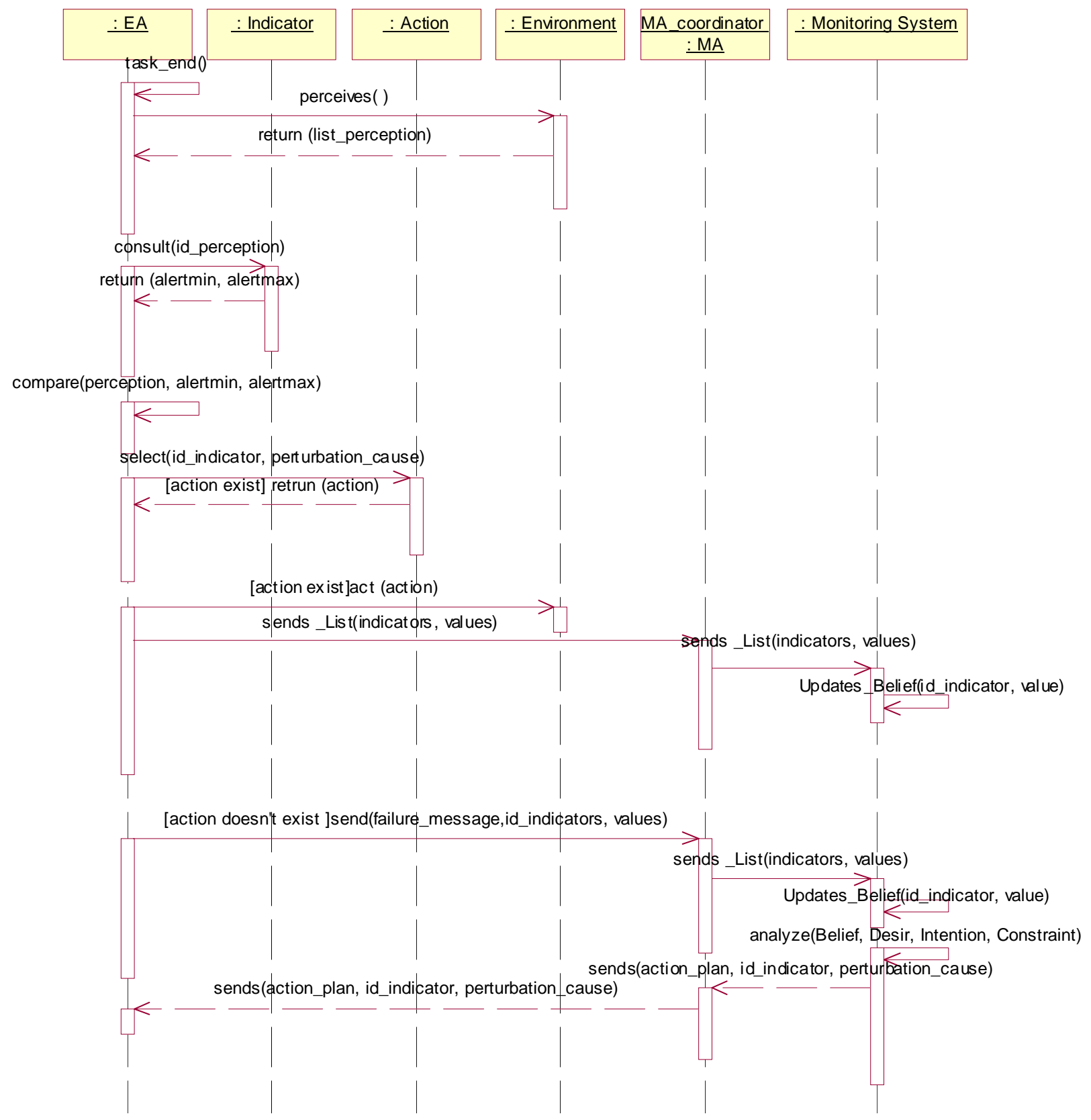

Fig 12. Sequence diagram of the monitoring and control protocol

\section{The implementation phase}

This section describes the last phase of the ArchMDE development process that highlights the transition from the modeling phase through the implementation one. This phase is essentially divided into two steps. The first one concerns the refinement of the agentified domain metamodel by integrating the information necessary for the implementation. The second step introduces the choice of the development platform. The next section focuses on both steps of the implementation phase of the ArchMDE development process. 


\section{Refinement of the agentified domain metamodel}

The steps of protocols integration identify the abstract patterns that describe the dynamic behavior of the agentified domain concepts. The refinement consists in the integration of the attributes and the methods that define the architectural and the behavioral properties of each concept. This step provides a final class diagram corresponding to the "Implementation Metamodel". The encoding of this metamodel leads to a dedicated simulation platform.

In the implementation metamodel, we have refined the domain metamodel and integrated the multiagent concepts that are essential to the agent's behavior and that do not have a counterpart during the agentification process.

Figure 13 represents the "Implementation Metamodel". The class diagram focuses on the main methods and attributes of the metamodel. Each private attribute has an accessor (get) and a mutator (set) method. However, these methods are not visible in order to reduce the pattern. The "Actor" class defines the overall architecture and behavior of an agent. The EA (reactive agent) and MA (cognitive agent) inherit the common characteristics from the "Actor" class. Nevertheless, each one implements the method "run" that describes its behavior and the method "HandleMessage(Message M)" allowing it to read and to construe the received message.

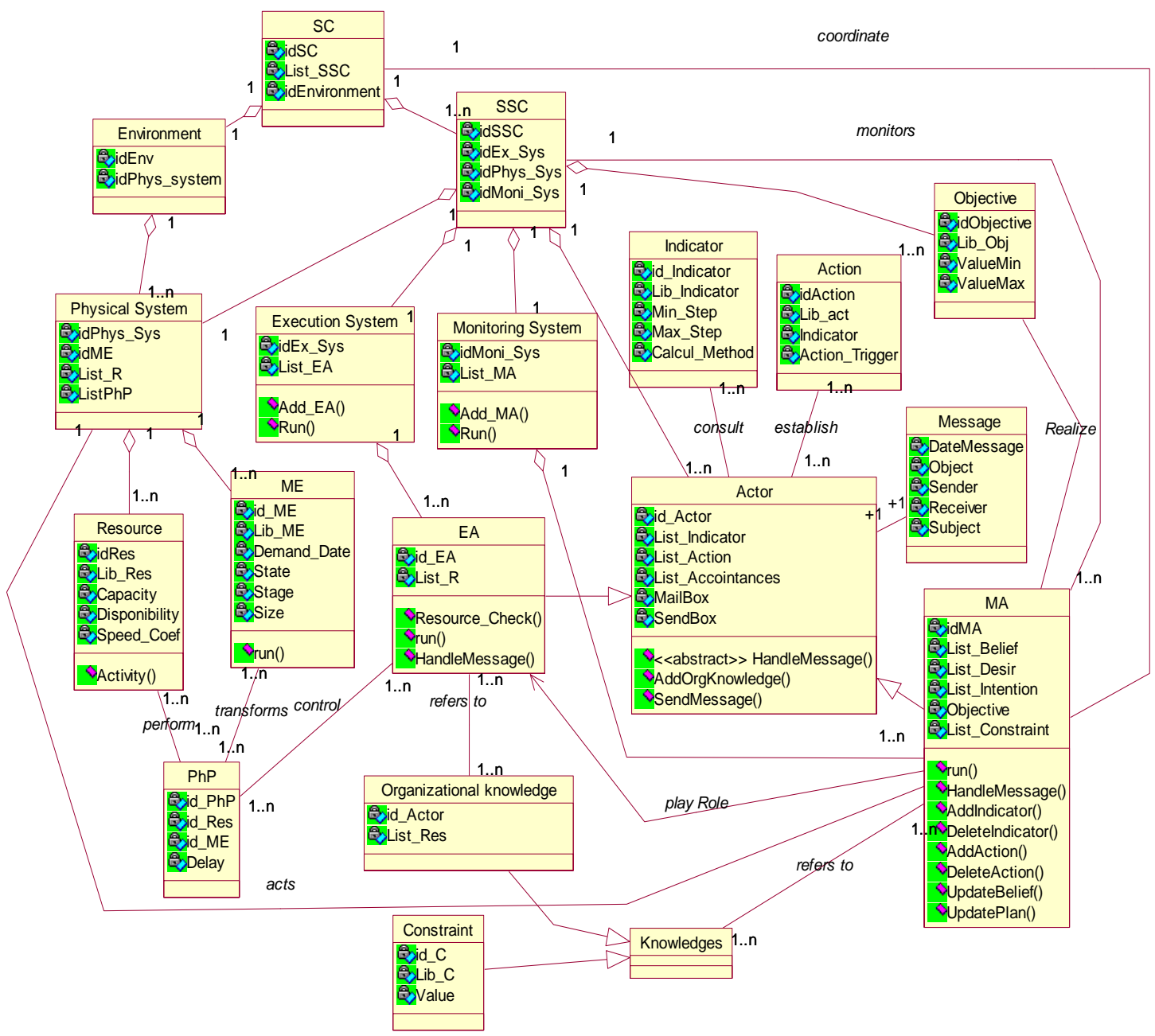

Fig 13. The Implementation metamodel 


\section{The choice of the development platform}

This section focuses on the choice of a development platform according to several objectives of the implementation. Indeed, obtaining the final pattern ("the implementation metamodel") completes the modeling process. At this level, the behavioral and architectural characteristics of the proposed concepts are implemented using a development platform. Below, we list the most important modeling criteria that the implementation platform must allow: (i) the implementation of cognitive agents (MA) and reactive agents (EA), (ii) the communication between agents (sending messages), (iii) the integration of the agents group (Monitoring System and Execution System) and the modeling of the external environment (Physical System), (iv) and a graphical interface for easy setup and the observation of the simulation results.

According to these criteria, we have studied two multiagent platforms: Jade (http://jade.cselt.it) and Madkit (http://www.madkit.net).

Jade (Java Agent Development Platform) is a multiagent platform fully encoded in JAVA. It allows the modeling of agents based on predefined patterns communicating through messages (FIPA-ACL). The Jade software simplifies the implementation through a graphical user interface (remote GUI). However, it does not allow the implementation of a group of agents which is a major modeling criterion. Consequently, Jade does not correspond to our specifications.

Madkit is a modular and scalable multiagent platform also written in JAVA. The main reasons for taking an interest in this software are that it: (i) provides an API (Application Programming Interface) to enable the development of agents that communicate through sending messages, (ii) allows one to develop agents situated in groups and play roles in the organization and (iii) offers a full set of facilities for launching, displaying, developing and monitoring agents and organizations (Gutknecht et al., 2000). But, Madkit does not allow one to draw the external environment as a set of objects (object in oriented-object programming theory). Indeed, each concept must be an agent in order to communicate in the application. However, we can use the Madkit platform with a Java environment to integrate external classes. So, Madkit merged with Java environment can be considered as an implementation way. Due to a lack of time and knowledge in this area, this solution can be considered as a future perspective to implement the knowledge model.

Given our computer skills, we have decided to implement the simulation platform using JAVA. In this platform, we have developed agents, groups of agents and structured a peer-to-peer communication between them. The elements external to the agent (i.e.: resources, indicator, action, $\mathrm{PhP}$, etc.) are encoded as objects. The product (ME) is an active entity. It is encoded as an object that triggers events.

\section{Industrial case study}

This part is a compatibility verification of the proposed metamodel with the Savoie industrial environment. The case study presented here deals with a SMEs group that acts as mechatronic providers in France and the Benelux countries. The products are manufactured in factories in China and Thailand. Then, they are distributed worldwide by providers such as the one studied. The production process is not visible to providers. Indeed, in this example, customer needs (forecasts and demands) are gathered annually by decision-making actors in the network. After 
that, the forecasts are sent to other organizations involved in production planning. The visibility in this supply chain is defined and limited by the geographical location.

In order to be consistent with the metamodel presented earlier, we consider the example of this supply chain as a node between several SSCs. Let us call this SMEs network 'SSC-France' and the other networks 'SSC-related country'. Thus, we get a model of the overall supply chain which is reflected in Figure 14.



Fig 14. Overall supply chain model of "SSC-France"

The focus is made on "SSC-France". In this insight, the first step concerns the study of the internal structure of the SSC-France according to the metamodel. Firstly, we have identified actors depending on their role and level of decision-making. Secondly, the resources that are needed were listed. Thirdly, the processes that are supported by SSC-France have been studied and sorted into the previously presented categories (see Table1). After that, each management process is studied in order to identify the data needed for actors to achieve their task (indicators and actions). The environment analysis is not presented here but details could be found in Tounsi, 2009.

The second steps deals with the instantiation of the final metamodel (Fig 13.). In fact, information collected (about actors, resources and processes) must be connected with a concept from the final metamodel. Table 4 summarizes the identification and the instantiation of the different actors in SSC-France and their resources.

Table 4. Identification and Instantiation of the actors and their resources

\begin{tabular}{|c|c|c|c|c|c|}
\hline \multicolumn{3}{|c|}{ Actors } & \multicolumn{3}{|c|}{ Resources } \\
\hline Function & $\begin{array}{c}\text { Name in } \\
\text { the model }\end{array}$ & $\begin{array}{c}\text { « Instance } \\
\text { of » }\end{array}$ & Function & $\begin{array}{c}\text { Name in the } \\
\text { model }\end{array}$ & $\begin{array}{c}\text { "Instance } \\
\text { of » }\end{array}$ \\
\hline Logistician & L1, L2, L3 & MA & - & - & - \\
\hline \multirow{3}{*}{ Storehouse } & \multirow[t]{3}{*}{ S1 } & \multirow[t]{3}{*}{ MA } & Warehouse & Ent_1 & \multirow{8}{*}{ 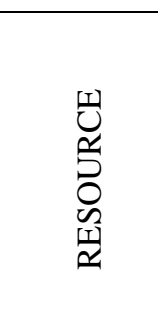 } \\
\hline & & & Receptionist & Rec_1 & \\
\hline & & & Order's picker & Pre_1 & \\
\hline \multirow{3}{*}{$\begin{array}{l}\text { Secondary } \\
\text { Storehouse }\end{array}$} & \multirow[t]{3}{*}{ S2 } & \multirow[t]{3}{*}{ EA } & Warehouse & Ent_2 & \\
\hline & & & Receptionist & Rec_2 & \\
\hline & & & Order's picker & Pre_2 & \\
\hline Maritime Carrier & $\mathrm{C} 1$ & EA & Boat & B_1 & \\
\hline Carrier & $\mathrm{C} 2$ & EA & Truck & B_2 & \\
\hline
\end{tabular}


Table5 describes the instantiation of the physical processes. Indeed, in the environment study of the SSC-France, three macro processes were identified as well as their corresponding elementary physical process.

Table 5. Identification and Instantiation of the physical processes

\begin{tabular}{|c|c|c|c|c|c|}
\hline \multicolumn{3}{|c|}{ Macro physical processes } & \multicolumn{3}{|c|}{ Elementary physical processes } \\
\hline Name & $\begin{array}{l}\text { Name in } \\
\text { the model }\end{array}$ & $\begin{array}{c}\text { « Instance } \\
\text { of » }\end{array}$ & Name & $\begin{array}{c}\text { Name in the } \\
\text { model }\end{array}$ & $\begin{array}{c}\text { « Instance } \\
\text { of » }\end{array}$ \\
\hline \multirow[t]{2}{*}{ Distribution } & \multirow[t]{2}{*}{ PhP_dis } & \multirow[t]{2}{*}{$\mathrm{PhP}$} & Order preparation & Op_dis_1 & $\mathrm{PhP}$ \\
\hline & & & Expedition & Op_dis_2 & $\mathrm{PhP}$ \\
\hline \multirow[t]{3}{*}{ Storage } & \multirow[t]{3}{*}{ PhP_stock } & \multirow[t]{3}{*}{$\mathrm{PhP}$} & $\begin{array}{l}\text { Transport } \\
\text { Asia/Marseille }\end{array}$ & Op_stock_1 & $\mathrm{PhP}$ \\
\hline & & & $\begin{array}{l}\text { Transport } \\
\text { Marseille/warehouse }\end{array}$ & Op_stock_2 & $\mathrm{PhP}$ \\
\hline & & & Reception and storage & Op_stock_3 & $\mathrm{PhP}$ \\
\hline \multirow{2}{*}{$\begin{array}{l}\text { Return } \\
\text { Management }\end{array}$} & \multirow[t]{2}{*}{ PhP_ret } & \multirow[t]{2}{*}{$\mathrm{PhP}$} & Return of product & Op_ret_1 & $\mathrm{PhP}$ \\
\hline & & & Delivery for exchange & Op_ret_2 & $\mathrm{PhP}$ \\
\hline
\end{tabular}

Table 6 highlights the main indicators and the related actions in the case of disturbance of the system. Based on the literature, we have proposed these indicators in order to evaluate the group performance. At time "T", the disturbance is observed when the value calculated of the indicator $\left(\mathrm{V}_{(\mathrm{I})}\right)$ is not in the right interval. This interval is defined by the concept "objective" with the attributes Ind_step_min and Ind_step_max.

Table 6. Identification and instantiation of the indicators and actions

\begin{tabular}{|c|c|c|c|c|}
\hline \multirow{2}{*}{$\begin{array}{c}\text { PhP } \\
\text { Name } \\
\end{array}$} & \multicolumn{2}{|c|}{ Indicator } & \multicolumn{2}{|c|}{ Action } \\
\hline & Name & Arithmetic Method & Name & Evaluation \\
\hline \multirow[t]{2}{*}{ Op_dis_1 } & $\begin{array}{l}\text { Time of order } \\
\text { preparation } \\
\text { (Tps_prep_comm) }\end{array}$ & $\begin{array}{l}\text { Time of order } \\
\text { preparation / the } \\
\text { average time of order } \\
\text { preparation }\end{array}$ & $\begin{array}{l}\text { Reorganization } \\
\text { (act_prep_comm) }\end{array}$ & $\begin{array}{l}\mathrm{V}_{(\mathrm{I})} \cdot \text { calculated }> \\
\text { Ind_step_max }\end{array}$ \\
\hline & $\begin{array}{l}\text { Rate of right order } \\
\text { (Tx_comm_just) }\end{array}$ & $\begin{array}{l}\text { Nbr of right orders / } \\
\text { Nbr of orders }\end{array}$ & $\begin{array}{l}\text { Training } \\
\text { (act_comm_just) }\end{array}$ & $\begin{array}{l}\mathrm{V}_{(\mathrm{I})} \cdot \text { calculated }< \\
\text { Ind_step_min }\end{array}$ \\
\hline \multirow[t]{2}{*}{ Op_dis_2 } & $\begin{array}{l}\text { Rate of order } \\
\text { respect date } \\
\text { (tx_resp_date) }\end{array}$ & $\begin{array}{l}\text { Nbr of orders at time } \\
\text { / nbr of orders }\end{array}$ & Training & $\begin{array}{l}\mathrm{V}_{(\mathrm{I})} \cdot \text { calculated }< \\
\text { Ind_step_min }\end{array}$ \\
\hline & $\begin{array}{l}\text { Rate of warehouse } \\
\text { load } \\
\text { (Tx_remplissage) }\end{array}$ & $\begin{array}{l}\text { Volume of used } \\
\text { storage / Volume of } \\
\text { storage }\end{array}$ & $\begin{array}{l}\text { Refer to the } \\
\text { Monitoring } \\
\text { System }\end{array}$ & In all cases \\
\hline $\begin{array}{l}\text { Op_Stock_1 } \\
\text { Op_Stock_2 }\end{array}$ & $\begin{array}{l}\text { Rate of damaged } \\
\text { product } \\
\text { (Tx_degrad) }\end{array}$ & $\begin{array}{l}\text { Value of damaged } \\
\text { products / Storage } \\
\text { value }\end{array}$ & $\begin{array}{l}\text { Refer to the } \\
\text { Monitoring } \\
\text { System }\end{array}$ & In all cases \\
\hline \multirow[t]{3}{*}{ Op_stock_3 } & $\begin{array}{l}\text { Reception date } \\
\text { (delai) }\end{array}$ & Date & $\begin{array}{l}\text { Available staff } \\
\text { (act_aug_personn } \\
\text { el) }\end{array}$ & $\begin{array}{l}\mathrm{V}_{(\mathrm{I})} \cdot \text { calculated }< \\
\text { Ind_step_min }\end{array}$ \\
\hline & $\begin{array}{l}\text { Rate of warehouse } \\
\text { load } \\
\text { (tx_remplissage) }\end{array}$ & $\begin{array}{l}\text { Volume of used } \\
\text { storage / Volume of } \\
\text { storage }\end{array}$ & $\begin{array}{l}\text { Refer to the } \\
\text { Monitoring } \\
\text { System }\end{array}$ & In all cases \\
\hline & $\begin{array}{l}\text { Inventory turnover } \\
\text { (tx_rot_stock) }\end{array}$ & $\begin{array}{l}\text { Number of orders / } \\
\text { the annual average } \\
\text { inventory }\end{array}$ & $\begin{array}{l}\text { Refer to the } \\
\text { Monitoring } \\
\text { System }\end{array}$ & In all cases \\
\hline
\end{tabular}


Figure 15 draws the "SSC-France" model which is an instantiation of the implementation metamodel based on the previous tables (Table 3, Table 4 and Table 5).

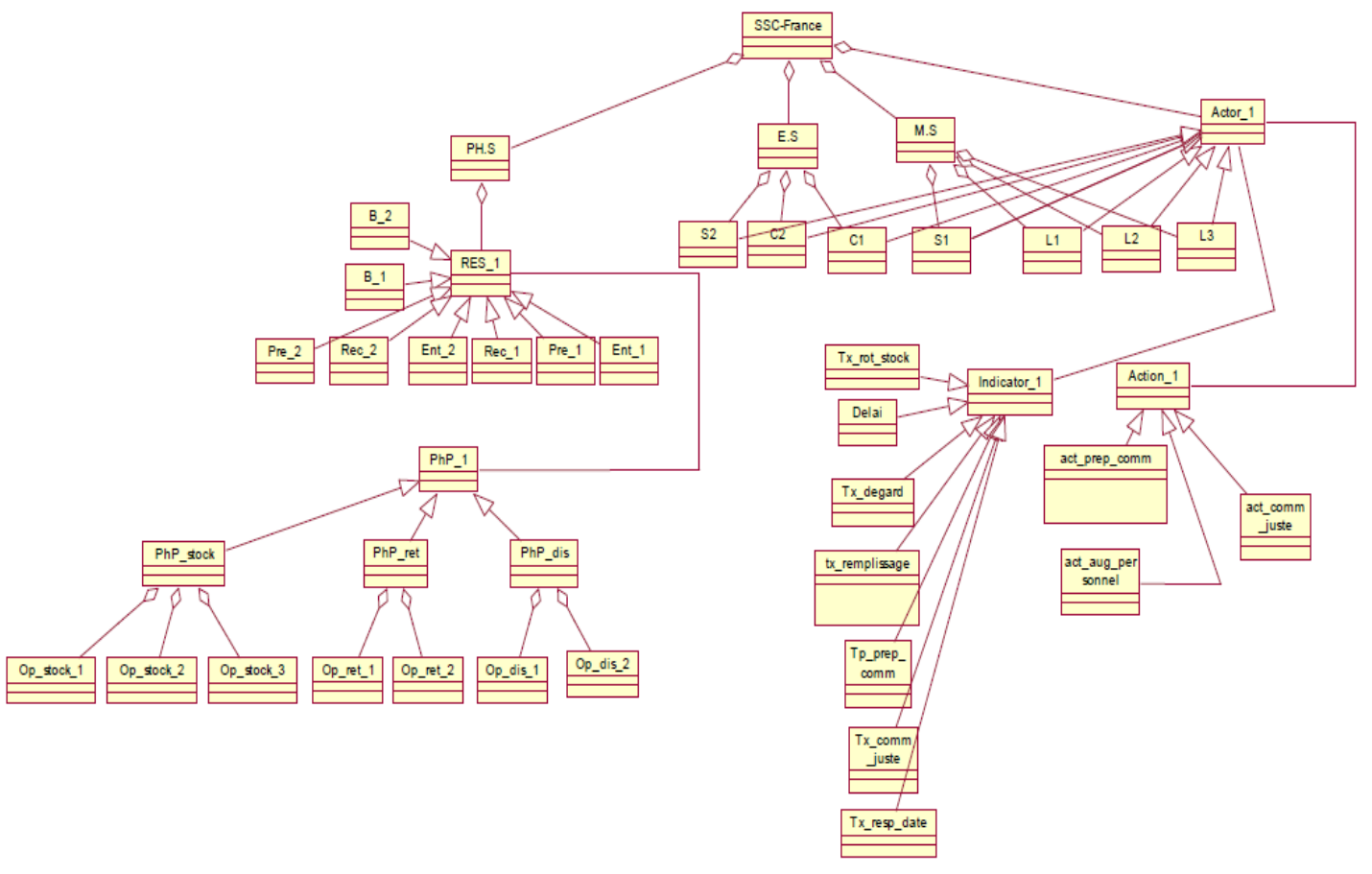

Fig 15. "SSC-France” model

\section{Conclusion and perspectives}

The research presented in this paper focuses on integrating SMEs mechatronic clusters in the overall supply chain modeling for simulation. The main objective of this work is to capitalize the know-how techniques in order to simplify the supply chain modeling and the concepts re-use. Hence, the paper proposes an agentified knowledge-model; the development process used to build this model up is the ArchMDE framework (Tounsi et al., 2009c) which separates the research area (context) from the computer modeling tool. This choice is motivated by the will to follow an approach that reliably describes the research context. This approach leads to develop the domain concepts with the possibility to re-use them in other research projects adopting different modeling tools.

According to this approach, the first phase of this work focuses on the study and the analysis of the research domain namely SMEs mechatronic supply chain. Then, concerning the domain, our main contributions are firstly the methodology adopted to generate the concepts of the domain studied and secondly its implementation (i.e. the domain metamodel). Hence, an overview of the different visions for supply chain design has enabled us to propose a methodology that combines the theories of the product vision, the structure vision and the process vision. Following this step, we apply the methodology framework to conceptualize the SMEs mechatronic supply chain. This implementation leads to a domain metamodel which describes the structural and architectural characteristics of the studied context.

The second phase highlights the computer modeling of the domain metamodel based on the multiagent system paradigm. In this phase, our contribution reflects the agentification of the 
domain concepts in order to study the behavior of the SMEs supply chain. In fact, we introduce a metamodel describing the architectural characteristics of multiagent systems. Thus, the agentification of the domain is built according to the natural analogy between the supply chain systems and those of multiagent. A description of the agentified domain concepts behavior is drawn by defining the interaction protocols. These protocols that have been developed allow the synchronization of the physical environment and the monitoring of the whole system (i.e. SMEs mechatronic supply chain).

In the last phase, we highlight the development of the future simulation platform resulting from the proposed knowledge-model and we describe how we can apply it on the industrial case.

The perspectives of this research work can be formulated in several areas. Within a short time, a major perspective to be addressed is to achieve the encoding of the simulation platform (currently in progress) in order to validate the knowledge-model with the simulation of case studies. In addition, integrating the optimization and the knowledge management in the decisional layer (i.e Monitoring System) are important aspects to implement.

In the future, this research work can be subject to the integration of other kinds of indicators or to the study and implementation of other interaction protocols.

\section{References}

[1] Al-Mutawah K., Lee V., and Cheung Y., 2009. A new multi-agent system framework for tacit knowledge management in manufacturing supply chains. Journal of Intelligent Manufacturing, vol. 20, N 5, pp. 593-610.

[2] Azaiez S., 2007. Approche Dirigée par les modèles pour le développement de systèmes multi-agents. Thèse de l’Université de Savoie, Spécialité Informatique. December 11, Annecy le vieux, France.

[3] Azaiez S., Habchi G., Huget M.P., Pralus M. and Tounsi J., 2007. Multiagent oriented modelling and simulation for manufacturing systems control. INDIN 2007, $5^{\text {th }}$ IEEE International Conference on Industrial Informatics, July 23-27. Vienna, Austria.

[4] Bagchi S., Buckley S., Ettl M., Lin G., 1998. Experience using the IBM supply chain simulator. Winter Simulation Conference.

[5] Beamon B.M, 1998. Supply chain designs and analysis: models and methods. International Journal of Production Economics, vol. 55, pp. 281-294.

[6] Berger T., Sallez Y., Valti B., Gibaud A., and Trentesaux A., 2010. Semi-heterarchical allocation and routing processes in FMS control: a stigmergic approach. Journal of Intelligent \& Robotics Systems, vol. 58, N¹, pp. 17-45.

[7] Bratman M.E, Israel D.J., Pollack M., 1998. Plans and resource-bounded practical reasoning. Computational Intelligence, 4, pp 349-355.

[8] Brooks R.A., 1991. Intelligence without representation. Artificial Intelligence, 47: 139-160.

[9] Chopra S. and Meindl P., 2001. Supply Chain Management: strategy planning and operation. Upper Saddle River, NJ: Prentice-hall.

[10] Cooper M., Lambert D.M. and Pagh J.D., 1997. Supply chain management: more than a new name for logistics. International Journal of Logistics Management, vol 18, $\mathrm{n}^{\circ} 2$, pp. 1-13.

[11] Demazeau Y., 1996. “Vowels”, Invited lecture, IWDAIMAS96.

[12] Drucker P.F., 1998. Management's new paradigms. Forbes, October, pp. 152-177.

[13] Fischer K., Müller J.P., Pischel M., 1995. Unifying control in layered agent architecture. IJCAI95, Agent Theory, Architecture and language workshop, pp 240-252

[14] Frayret J.M, D’amours S., Rousseau A., Harvey S., et Gaudreault J., 2008. Agent-based supply chain planning in the forest products industry. International Journal of Flexible Manufacturing Systems, vol. 19, n²4, pp. 358-391.

[15] Fung R.Y.K et Chen T., 2005. A multiagent supply chain planning and coordination architecture. The International Journal of Advanced Manufacturing Technology, vol. 25, n 7-8, pp. 811-819.

[16] Gutknecht O., Ferber J. et Michel F., 2000. MadKit: Une expérience d'architecture de plate-forme multi-agents générique. $8^{\text {ème }}$ Journées Francophones Intelligence Artificielle Distribuée Systèmes Multi-Agents JFIADSMA 2000. Saint-Jean Le Vêtre Octobre.

[17] Ingalls R.G, 1998. The value of simulation in modeling supply chain. In Proceedings of the 1998 winter simulation conference, Washington DC, pp. 1371-1375.

[18] Janssen M., 2005. The architecture and business value of a semi-cooperative, agent-based supply chain management system. Electronic Commerce Research and Applications (4), pp. 315-328.

[19] Julien P.A., 1997. Les PME bilan et perspectives. $2^{\mathrm{e}}$ edition, Economica, Paris, France.

[20] Kent S., 2002. Model-driven Engineering, IFM 2002, vol. 2335 of LNCS, Springer-Verlag, pp. 286-298.

[21] Labarthe O., Espinasse B., Ferrarini A., Montreuil B., 2007. Toward a methodological framework for agent-based modeling and simulation of supply chains in a mass customization context. Simulation Modeling Practice and Theory. 15, pp.113-136

[22] Lambert D.M. and Cooper M.C., 2000. Issues in supply chain management. Industrial Marketing Management, 29, $\mathrm{n}^{\circ}$ 1, pp. 65-83. 
[23] Lee Y.H, Cho M.K., Kim S.J. and Kim Y.B, 2002. Supply chain simulation with discrete continuous combined modelling. Computer and Industrial Engineering, 43, pp 375-392.

[24] Longo F. And Mirabelli G., 2008. An advanced supply chain management tool based on modelling and simulation. Computers \& Industrial Engineering, vol 54, pp. 570-588.

[25] Monostori T., Vancza J. and Kumara S.R.T, 2006. Agent-based systems for manufacturing. Annals of the CIRP, vol. 55, n², pp. 697-720.

[26] Monteiro T., Anciaux D., D’amours S., Espinasse B., Ferrarini A., Labarthe O., Montreuil B. and Roy D., 2008. Simulation à base d'agents des systèmes de coordination et de planification des réseaux d'entreprises. Chapitre 7 de : La simulation pour la gestion des chaînes logistiques, Lavoisier, Hermes Science Publications.

[27] Oztemel E. and Tekez E.K. Interaction of agents in performance based supply chain management. Journal of Intelligent Manufacturing, vol. 20, $\mathrm{N}^{\circ} 2$, pp.159-167.

[28] Parunak H., Savit R., Riolo R.L, 1998(a). Agent-based modelling VS equation-based modelling: a case study and user's guide. Proceedings of Multi-agent systems and agent-based simulation (MABS'98), Springer, LNAI 1534.

[29] Parunak H., 1998. Wat can agents do in industry, and why? An overview of industrially-oriented R\&D at CEC. Second international workshop on cooperative information agents, CIA'98, M.Klusch.

[30] Parunak H.V.D, 1999. Industrial and practical applications of DAI. In G. Weiss (ed), Multi-agents systems, MIT Press.

[31] Parunak H., Savit R., Riolo R., and Stevens J., 1999. DASCh : Dynamic analysis of supply chains. Center of Electronic Commerce, DASCh final Report, part 0: Executive Summary.

[32] Sarimveis H., Patrinos P., Tarantilis C.D and Kiranoudis C.T, 2008. Dynamic modelling and control of supply chain systems: A Review. Computers \& Operations Research, 35, pp. 3530-3561.

[33] Shen W., et Norrie D.H., 1999. Agent-Based Systems for Intelligent Manufacturing : A State-of-the-Art Survey. Knowledge and Information Systems, an International Journal, vol.1, n², pp.129-156.

[34] Shen W., Hao Q., Yoon H.J, et Norrie D.H., 2006. Applications of agent-based systems in intelligent manufacturing: an updated review. Advanced Engineering Informatics, vol. 20, pp. 415-431

[35] Stevens G.C., 1989. Integrating the supply chain. International Journal of Physical Distribution and Materials Management, 19, pp 3-8.

[36] Terzi S. and Cavalieri S., 2004. Simulation in the supply chain context: a survey. Computers in Industry, 53, pp.3-16.

[37] Thierry C. 2003. Gestion des chaînes logistiques : Modèle et mise en œuvre pour l'aide à la décision à moyen terme. Accreditation to supervise research. University of Toulouse II.

[38] Tounsi J., Boissière J., Habchi G., 2008. A conceptual model for SME Mechatronics supply chain. $6^{\text {th }}$ International Industrial Simulation Conference (ISC’08), Lyon, France, pp. 273-280.

[39] Tounsi J., 2009. Modélisation pour la simulation de la chaîne logistique globale dans un environnement de production PME mécatronique. PhD Thesis of The University of Savoie, Industrial Engineering, $4^{\text {th }}$ of December 2009.

[40] Tounsi J., Boissière J, Habchi G., 2009a.Multiagent decision making for SME supply chain simulation. In Proceedings of 23rd European Conference on Modeling and Simulation (ECMS), pp. 203-211, Madrid, Espagne.

[41] Tounsi J., Habchi G., Boissière J., 2009b. A multiagent system for production synchronization in SME mechatronic supply chain. In Proceedings of the 10th Middle Eastern Simulation and Modeling Conference (MESM), pp. 91- 97, Beirut, Liban.

[42] Tounsi J., Azaiez S., Habchi G., Boissière J., 2009c. A multiagent approach for modelling SMEs mechatronic supply chains. In Proceedings of the $13^{\text {th }}$ IFAC Symposium on Information Control Problems in Manufacturing.

[43] Villarreal Lizarraga, C.L., Dupont L., Gourg D., Pingaud H., 2005. Contributing to management of shared projects in SMEs manufacturing clusters. $18^{\text {th }}$ International Conference on Production Research (ICPR-18), Salerno, Italy.

[44] Wooldridge M., 1999. Intelligent Agents. Multiagents Systems, Weiss G., Ed:MIT Press.

[45] Wooldridge M., 2002. An introduction to Multiagent Systems. Jonh Wiley \& Sons, February.

[46] Yuan Y., Liang T.P and Zhang J.J, 2002. Using agent technology to support supply chain management: potentials and challenges. Mc Master University. 